\title{
On the road to the Preliminary Design Review of the MAORY adaptive optics module for ELT
}

Emiliano Diolaiti*a ${ }^{\mathrm{a}}$, Paolo Ciliegi ${ }^{\mathrm{a}}$, Renata Abicca ${ }^{\mathrm{a}}$, Guido Agapito ${ }^{\mathrm{b}}$, Carmelo Arcidiacono ${ }^{\mathrm{a}}$, Andrea Baruffolo $^{c}$, Michele Bellazzini ${ }^{\mathrm{a}}$, Valdemaro Biliotti ${ }^{\mathrm{b}}$, Marco Bonaglia ${ }^{\mathrm{b}}$, Giovanni Bregoli ${ }^{\mathrm{a}}$, Runa Briguglio $^{\mathrm{b}}$, Olivier Brissaud ${ }^{\mathrm{d}}$, Lorenzo Busoni ${ }^{\mathrm{b}}$, Luca Carbonaro ${ }^{\mathrm{b}}$, Alexis Carlotti ${ }^{\mathrm{d}}$, Enrico Cascone $^{\mathrm{e}}$, Jean-Jacques Correia ${ }^{\mathrm{d}}$, Fausto Cortecchia ${ }^{\mathrm{a}}$, Giuseppe Cosentino ${ }^{\mathrm{f}}$, Vincenzo De Caprio ${ }^{\mathrm{e}}$, Marco de Pascale ${ }^{\mathrm{c}}$, Adriano De Rosa ${ }^{\mathrm{g}}$, Ciro Del Vecchio ${ }^{\mathrm{b}}$, Alain Delboulbéd ${ }^{\mathrm{d}}$, Gianluca Di Rico ${ }^{\mathrm{j}}$, Simone Esposito $^{\mathrm{b}}$, Daniela Fantinel ${ }^{\mathrm{c}}$, Philippe Feautrier ${ }^{\mathrm{d}}$, Corrado Felini ${ }^{\mathrm{e}}$, Debora Ferruzzi ${ }^{\mathrm{b}}$, Luca Fini $^{\mathrm{b}}$, Giuliana Fiorentino ${ }^{\mathrm{a}}$, Italo Foppiani ${ }^{\mathrm{a}}$, Mauro Ghigo ${ }^{\mathrm{h}}$, Christophe Giordano $^{\mathrm{b}}$, Enrico Giro ${ }^{\mathrm{c}}$, Laurence Gluck $^{\mathrm{d}}$, François Hénault ${ }^{\mathrm{d}}$, Laurent Jocou ${ }^{\mathrm{d}}$, Florian Kerber ${ }^{\mathrm{i}}$, Paolo La Penna ${ }^{\mathrm{i}}$, Sylvain Lafrasse $^{\mathrm{d}}$, Mimma Lauria ${ }^{\mathrm{b}}$, Etienne le Coarer ${ }^{\mathrm{d}}$, Miska Le Louarn ${ }^{\mathrm{i}}$, Matteo Lombini ${ }^{\mathrm{a}}$, Yves Magnard ${ }^{\mathrm{d}}$, Elisabetta Maiorano $^{\mathrm{g}}$, Filippo Mannucci ${ }^{\mathrm{b}}$, Michela Mapelli ${ }^{\mathrm{c}}$, Enrico Marchetti ${ }^{\mathrm{i}}$, Didier Maurel ${ }^{\mathrm{d}}$, Laurence Michaud $^{\mathrm{d}}$, Gianluca Morgante ${ }^{\mathrm{g}}$, Manuele Moschetti ${ }^{\mathrm{h}}$, Thibaut Moulin ${ }^{\mathrm{d}}$, Sylvain Oberti ${ }^{\mathrm{i}}$,

Giovanni Pareschi ${ }^{\mathrm{h}}$, Mauro Patti ${ }^{\mathrm{a}, \mathrm{f}}$, Cedric Plantet ${ }^{\mathrm{b}}$, Alfio Puglisi ${ }^{\mathrm{b}}$, Patrick Rabou ${ }^{\mathrm{d}}$, Roberto Ragazzoni $^{\mathrm{c}}$, Suzanne Ramsay ${ }^{\mathrm{i}}$, Armando Riccardi ${ }^{\mathrm{b}}$, Marco Riva ${ }^{\mathrm{h}}$, Sylvain Rochat ${ }^{\mathrm{d}}$, Frédéric Roussel $^{d}$, Alain Roux ${ }^{\mathrm{d}}$, Bernardo Salasnich $^{\mathrm{c}}$, Paolo Saracco ${ }^{\mathrm{h}}$, Laura Schreiber ${ }^{\mathrm{a}}$, Marilena Spavone ${ }^{\mathrm{e}}$, Eric Stadler ${ }^{\mathrm{d}}$, Marie-Hélène Sztefek ${ }^{\mathrm{d}}$, Noël Ventura ${ }^{\mathrm{d}}$, Christophe Vérinaud ${ }^{\mathrm{d}}$, Marco Xompero ${ }^{\mathrm{b}}$, Adriano Fontana ${ }^{1}$, Filippo Maria Zerbi $^{1}$

${ }^{a}$ INAF - Osservatorio Astronomico di Bologna, via Gobetti 93/3, 40129 Bologna, Italy;

${ }^{b}$ INAF - Osservatorio Astrofisico di Arcetri, largo E. Fermi 5, 50125 Firenze, Italy;

'INAF - Osservatorio Astronomico di Padova, vicolo dell'Osservatorio 5, 35122 Padova, Italy;

${ }^{\mathrm{d}}$ INSU - IPAG, 414 rue de la piscine, BP 53, 38041 Grenoble Cedex 9, France;

${ }^{\mathrm{e}} \mathrm{INAF}$ - Osservatorio Astronomico di Capodimonte, Salita Moiariello 16, 80131 Napoli, Italy;

${ }^{f}$ Università di Bologna - DIFA, via Gobetti 93/3, 40129 Bologna, Italy;

${ }^{g}$ INAF - Istituto di Astrofisica Spaziale e Fisica Cosmica, via Gobetti 101, 40129 Bologna, Italy;

${ }^{\mathrm{h}}$ INAF - Osservatorio Astronomico di Brera, via E. Bianchi 46, 23807 Merate, Italy;

${ }^{\mathrm{i}}$ European Southern Observatory, K.-Schwarzschild-Str. 2, 85748 Garching b. Muenchen, Germany;

${ }^{\mathrm{j}}$ INAF - Osservatorio Astronomico d'Abruzzo, via Maggini snc, 64100 Teramo, Italy;

${ }^{1}$ INAF - Istituto Nazionale di Astrofisica, viale del Parco Mellini 84, 00036 Roma, Italy

\begin{abstract}
The MAORY adaptive optics module will enable high angular resolution observations in the near infrared on the European ELT. This paper describes the current instrument design.
\end{abstract}

Keywords: Extremely Large Telescopes, ELT, multi-conjugate adaptive optics, LGS, MAORY

\section{INTRODUCTION}

MAORY $^{[1]}$ is one of the approved first-light instruments for the European Extremely Large Telescope (ELT) ${ }^{[2][3]}$. It is an adaptive optics module, enabling high-angular resolution observations in the near infrared by real-time compensation of the wavefront distortions due to the atmospheric turbulence and other disturbances such as the wind action on the telescope.

*emiliano.diolaiti@ oabo.inaf.it; phone +39 051 6357311; fax +39 051 6357390; www.oabo.inaf.it 
MAORY is designed to support the MICADO near-infrared camera ${ }^{[4]}$ by offering two adaptive optics modes: MultiConjugate Adaptive Optics (MCAO) and Single-Conjugate Adaptive Optics (SCAO).

The MCAO mode is required to achieve spatially uniform adaptive optics compensation over the scientific field of view with high sky coverage; wavefront sensing is performed by up to six artificial Laser Guide Stars (LGS) and up to three Natural Guide Stars (NGS), respectively for the measurement of high and low-order wavefront perturbations; wavefront compensation is performed by adaptive Deformable Mirrors (DMs) in MAORY, which work together with the telescope's adaptive and tip-tilt mirrors M4 and M5 respectively.

The SCAO mode is required to achieve peak adaptive optics performance on a limited field of view, when a sufficiently bright reference object is available nearby the scientific target; wavefront distortions are measured by a single NGS wavefront sensor and compensated by the telescope M4 and M5 mirrors, while the adaptive mirrors inside MAORY are kept fixed at their reference shape. The development of the SCAO mode is a joint endeavour between the MAORY and MICADO instrument teams.

MAORY also offers provision for a second port for a future instrument, as yet undefined.

As the project is progressing in its phase B towards the Preliminary Design Review, which is planned in 2018, a snapshot of the current design and expected performance of the instrument is presented in this contribution.

\section{INSTRUMENT ADAPTIVE OPTICS ARCHITECTURE}

\subsection{Wavefront control interface with the adaptive telescope}

The control equipment of the adaptive ELT maintains the telescope alignment at a level such that the optical quality is within the capture range of MAORY. Quasi-static aberrations in the telescope are compensated by the active optics system. Adaptive optics compensation of fast wavefront aberrations due to atmospheric turbulence, windshake effects etc. relies on resources distributed in MAORY (LGS and NGS wavefront sensors, post-focal DMs, real-time computer) and in the telescope (LGS launchers, adaptive quaternary mirror M4, tip-tilt mirror M5, other actuators such as the telescope main structure).

\subsection{MCAO mode}

The instrument sub-systems and their inter-relations in MCAO mode are shown in the functional block diagram in Figure 1. The MCAO mode of MAORY is based on the use of six LGS Wavefront Sensors (WFS) and up to three NGS wavefront sensors.

The light beam from the telescope, already pre-compensated by the actuators in the telescope, is propagated through the MAORY main path optics (common path). Upon wavefront compensation by the post-focal DMs, the light is split by a dichroic beam-splitter.

The light of wavelength shorter than about $600 \mathrm{~nm}$ is propagated from the dichroic beam-splitter to the LGS path optics (also known as LGS objective) and then to the LGS WFS sub-system. The light of wavelength longer than about $600 \mathrm{~nm}$ is propagated from the dichroic beam-splitter through the last segment of the main path optics (science path) to the exit port.

At the exit port, the MAORY focal plane is made available to MICADO, or another instrument in the case of the second port. The light of the NGSs is picked off by the Low-Order and Reference (LOR) WFS units in the NGS WFS module ${ }^{[5]}$. The LOR WFS serves different functions, among which: i) measuring the low-order modes (tip-tilt, but also focus and astigmatism) which are not reliably measured by the LGS WFS, due to the well-known tilt indetermination problem and to fast sodium layer instabilities; ii) providing a way to "de-trend" the low/medium order modes, other than tip-tilt, focus and astigmatism, which are affected by sodium layer temporal variations coupled to instrumental effects in the LGS WFS such as spot truncation ${ }^{[6]}$.

The wavefront measurements performed by the LGS and LOR WFSs are processed by the MAORY Real Time Computer (RTC), which drives in closed loop the post-focal DMs and, through the telescope Centralised Control System (CCS), also the actuators in the telescope, including the adaptive quaternary mirror M4 and the tip-tilt mirror M5.

All instrument operations are controlled and supervised by the MAORY instrumentation software, which also provides interfaces to the telescope CCS and to the MICADO (or other) client instrument instrumentation software. 


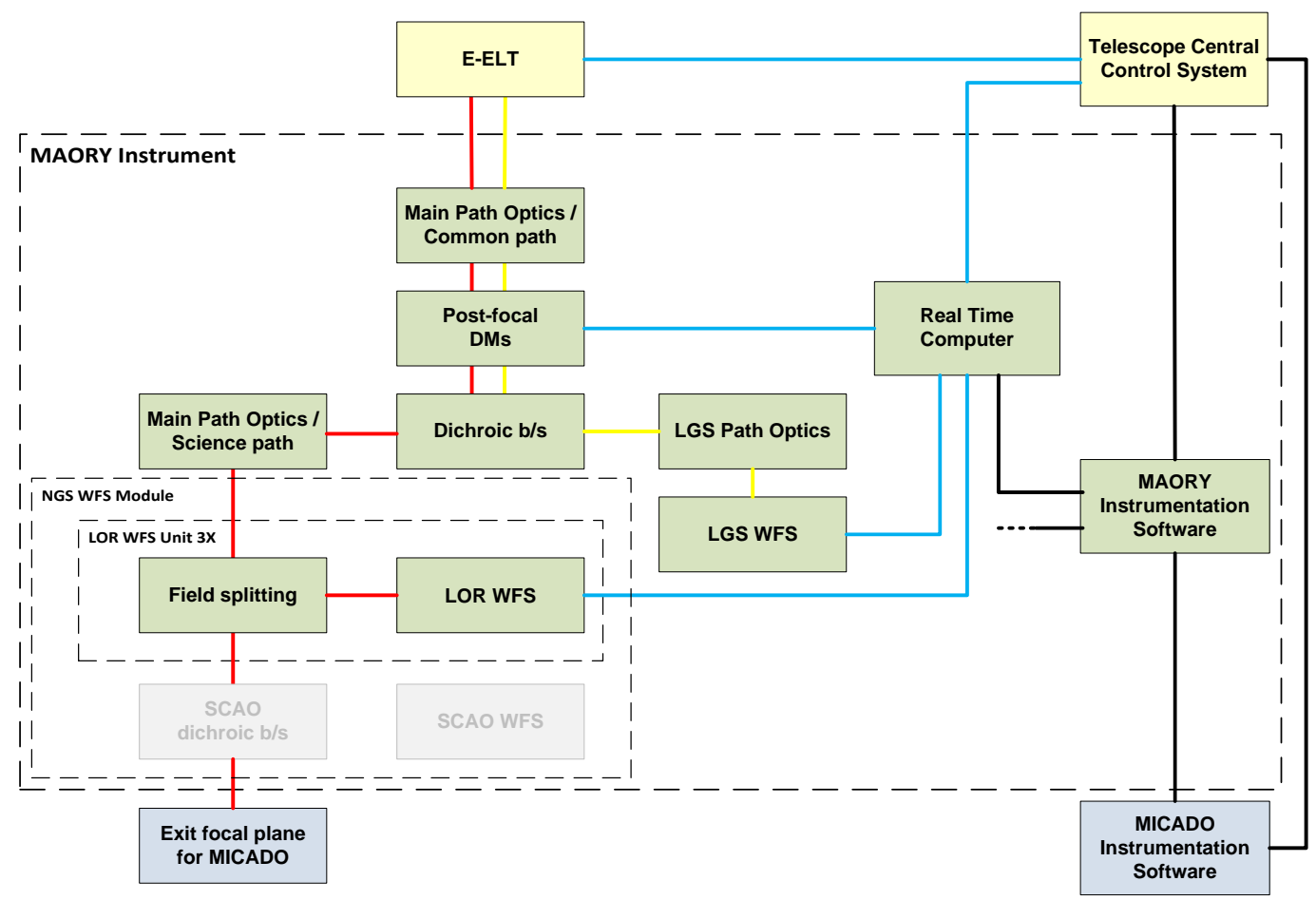

Figure 1. MCAO functional block diagram. Red lines: common and science light path. Yellow lines: LGS light path. Blue lines: real-time signals. Black lines: non real-time signals. Light grey blocks are not used in MCAO mode.

\subsection{SCAO mode}

In the SCAO mode ${ }^{[7]}$ the LGS and the LOR WFSs are not used. A dedicated SCAO WFS is used instead.

The light path is essentially the same as in the MCAO mode, with the exception of the exit port. Here the light of a NGS nearby the scientific target of interest is selected in wavelength by a deployable SCAO dichroic beam-splitter, which is inserted in the beam just upstream the exit focal plane. This SCAO dichroic beam-splitter transmits the science wavelengths to MICADO and reflects the wavefront sensing wavelengths to the SCAO WFS.

Signals from the SCAO WFS in the NGS wavefront sensor module are read by the MAORY RTC, which drives the actuators in the telescope through the CCS.

In the SCAO mode the MAORY post-focal DMs are kept at their reference shape as a baseline.

\section{INSTRUMENT DESIGN AND PERFORMANCE}

\subsection{Post-focal relay optics and main structure}

The post-focal relay optics sub-system of MAORY ${ }^{[8]}{ }^{[9]}$ re-images the telescope focal plane to the exit ports. The layout of the post-focal relay optics is shown in Figure 2 - left.

The sub-system contains the following channels:

- main path optics, which relay the telescope focal plane to the exit ports for the science instruments (MICADO and second instrument as yet undefined);

- $\quad$ LGS objective ${ }^{[10]}$, which creates an image plane for the LGS WFS sub-system. 
Light separation between the two channels is accomplished by the LGS dichroic beam-splitter, which splits the light according to wavelengths.

Inside the optical path, two clear planes are created, optically conjugated to two different ranges from the telescope entrance pupil, allowing the insertion of up to two DMs.

The main path optics design is based on six mirrors and the above mentioned LGS dichroic beam-splitter. In the baseline configuration, the beam-splitter is assumed to reflect the science path wavelengths: in this way, the path from the telescope focal plane to the instrument focal plane consists of reflecting optics only, nominally free from chromatic effects. The number of mirrors has been minimised to reduce thermal emissivity, as MAORY is not cooled as a baseline: the thermal background is therefore controlled by minimum number of optics and by suitable maintenance procedures to ensure optics cleanliness. Two mirrors are adaptive in the full instrument configuration; a partial implementation with 1 DM only is possible, with the second DM replaced by a rigid mirror.

The LGS objective design is based on both mirrors and lenses. The exit focal ratio is F/5, to reduce the focus range when the sodium layer distance changes with zenith distance during observations.

The main parameters of the main path optics and of the LGS objective are reported in the next tables.

Table 1. Main path optics parameters.

\begin{tabular}{|l|l|}
\hline Item & Value \\
\hline Exit focal ratio & F/17.7 (1:1 relay) \\
\hline Exit pupil distance & $8000 \mathrm{~mm}$ (towards telescope) \\
\hline Focal plane curvature & Flat \\
\hline NGS patrol FoV (also called technical FoV) & 200 arcsec diameter \\
\hline MICADO science FoV & Up to 75 arcsec diameter \\
\hline Transmitted wavelength range at exit port & $\begin{array}{l}0.6-2.5 \mu \mathrm{m} \\
\text { Lower wavelength limit set by LGS dichroic }\end{array}$ \\
\hline Post-focal DMs conjugation range & $15 \mathrm{~km}, 4.4 \mathrm{~km}$ \\
\hline Projected pitch of DMs on conjugates & $2 \mathrm{~m}$ \\
\hline
\end{tabular}

Table 2. LGS objective parameters.

\begin{tabular}{|l|l|}
\hline Item & Value \\
\hline Operating wavelength & $0.589 \mu \mathrm{m}$ \\
\hline LGS range to be re-imaged & $84-240 \mathrm{~km}$ \\
\hline Exit focal ratio & $\mathrm{F} / 5$ \\
\hline Exit pupil distance & Infinity \\
\hline LGS constellation maximum angular diameter & 120 arcsec \\
\hline
\end{tabular}

The exit port for MICADO is gravity invariant. The exit port for the second instrument is created by inserting a deployable flat mirror in the optical path; this port is not gravity-invariant.

The mechanical layout of the instrument is shown in Figure 2 - right. The post-focal relay optics is supported by the instrument main structure, which consists of a bench, connected to the Nasmyth platform through a support system made of four bipods in the current design, and a cover, which is aimed at creating an isothermal environment around the optics. 
A passive thermal concept is adopted. Daytime ventilation of the optics is planned, to help thermalisation by flushing cold air at the foreseen temperature for the night. During night-time, MAORY follows the ambient temperature.

MICADO is detached from MAORY and is supported by its own support system. Active and adaptive compensation of relative instabilities between the two instruments will be needed.

The last mirror of the MAORY main path optics is flat and folds the light down to MICADO in gravity invariant configuration. M11 is supported by a deployable swing arm, to allow mounting/dismounting operations of MICADO from above by the dome crane. This concept is currently under review: an alternative solution is to mount M11 directly on the fixed part of MICADO, thus avoiding the cantilevered part of the MAORY bench, which only has the purpose to support the MAORY cover around M11 and around MICADO. If this alternative concept will be adopted, the two instruments will have to be enclosed anyway inside a single cover to create a suitable environment for the optics.
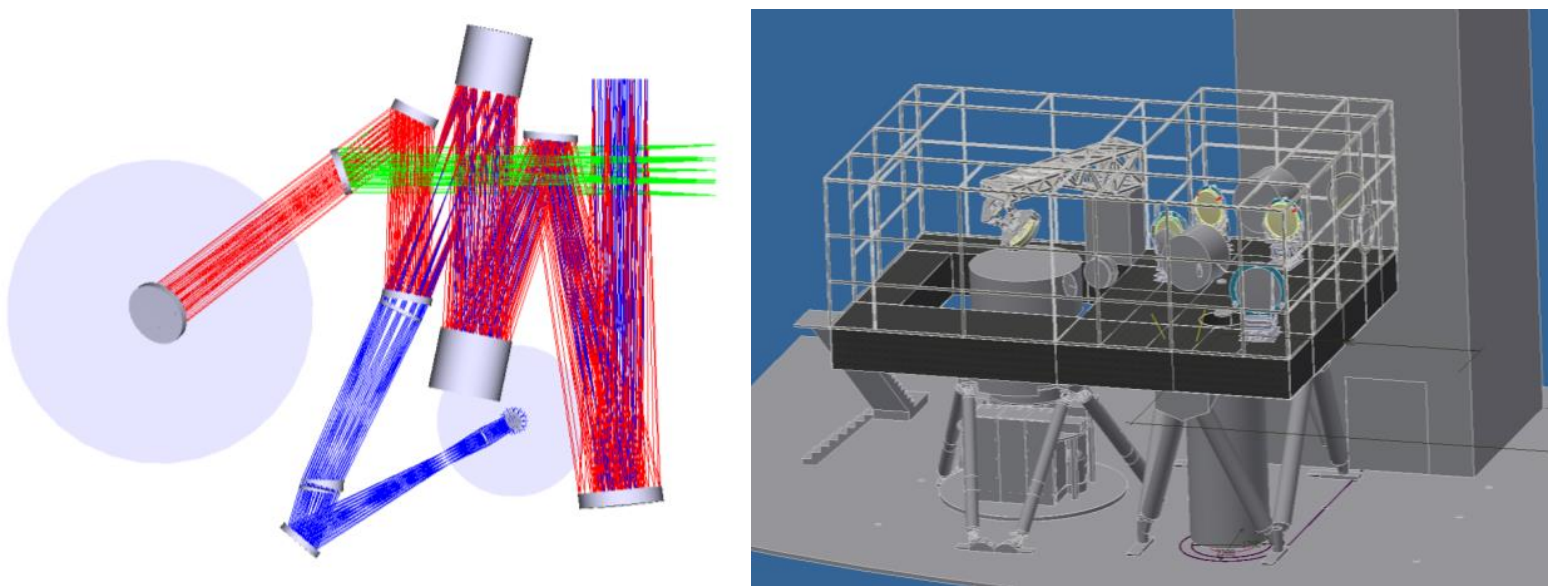

Figure 2. Left: post-focal relay optical layout. Red rays: light path to MICADO port; green rays: light path to second instrument port (achieved by deployable flat mirror); blue rays: LGS light path. Light grey circles represent the approximate volumes of MICADO and LGS WFS. Right: mechanical layout of MAORY on ELT Nasmyth platform. MICADO is also shown, on its independent support structure, besides the MAORY bench on the left.

\subsection{LGS wavefront sensor}

The opto-mechanical layout of the LGS WFS is shown in Figure 3. The sub-system is attached to the bottom plane of the MAORY bench in gravity-invariant configuration, to ensure stable mounting.

The LGS WFS consists of six WFS probes, one per LGS. The probes are mounted on a common focusing stage, to track the global sodium focus as the zenith angle changes during the observation, and on a common rotation stage, to track the rotation of the LGS asterism with the elevation axis, as the LGSs are launched fixed with respect to the telescope pupil. Each WFS probe is also provided with its own hexapod system for differential adjustments.

The images of the LGSs, produced by the LGS objective described in section 3.1, are selected by pick-off mirrors, which feed the six LGS WFS probes. The LGSs are launched 45 arcsec off-axis, as a baseline for the MICADO application. Larger angles are possible to ensure uniform compensation for a wide-field instrument on the second exit port.

The six WFS probes include a tip-tilt mirror each, for local jitter compensation. The jitter compensation function might also be implemented by the LGS launchers in the telescope, although the local jitter capability might be retained for calibration purposes.

The LGS WFS is based on a Shack-Hartmann configuration of order $80 \times 80$ sub-apertures. The baseline detector choice allows 10x10 pixels per subaperture. A trade-off study is in progress regarding the field of view and the spot sampling. Possible choices are 15 arcsec field of view with 1.5 arcsec/pixel sampling or 10 arcsec field of view with 1 arcsec/pixel sampling. The former reduces LGS spot truncation effects, relaxing the requirements on the NGS Reference WFS; the latter is better in terms of spot sampling. Mitigation strategies are under study to control the issues which are present in 
both cases. In particular, mitigation strategies for under-sampling effects are LGS spot blurring, if the LGS flux is high enough to ensure sufficient sensitivity, and WFS response calibration techniques by spot modulation. A mitigation strategy for spot truncation is the use of advanced tomographic schemes, weighting the slope measurements according to the elongation of the corresponding spots.
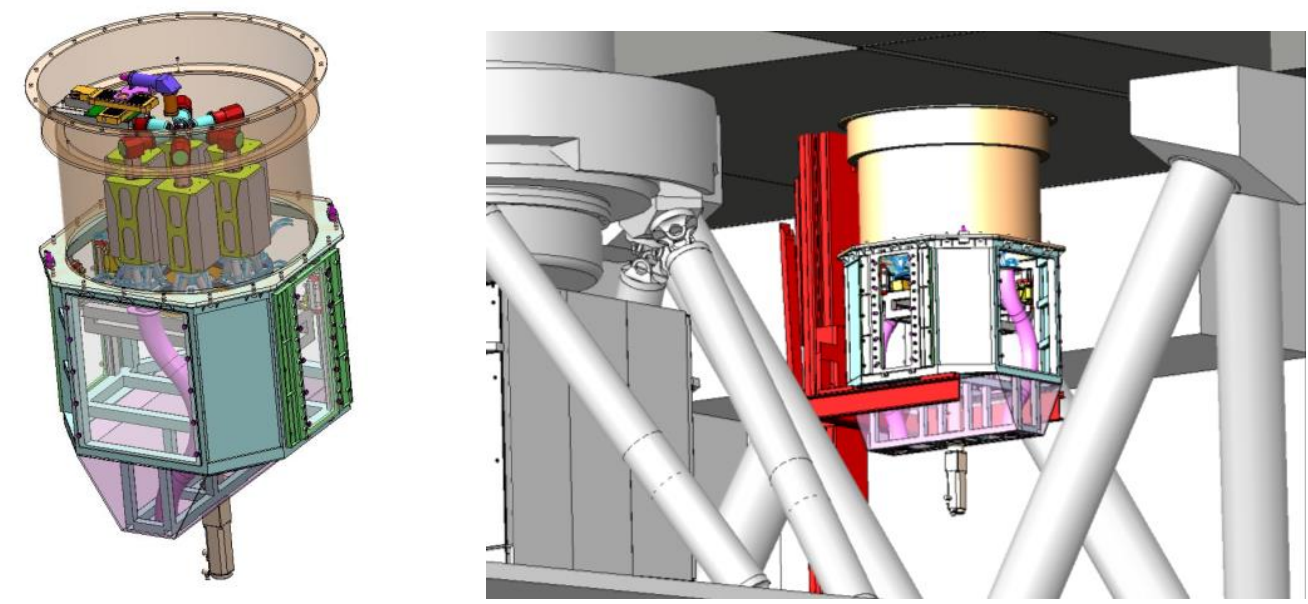

Figure 3. Left: LGS wavefront sensor opto-mechanical assembly. Right: the sub-system is attached to the bottom of the MAORY bench in gravity invariant configuration.

\subsection{NGS wavefront sensor module}

The opto-mechanical layout of the NGS WFS module ${ }^{[5]}$ is shown in Figure 4. This sub-subsystem is mounted on the MICADO cryostat, in order to minimise non-common path aberrations with the science instrument, in particular differential tip-tilt.

The NGS WFS module is divided in two parts:

- $\quad$ LOR WFS plate, holding the three WFS probes for the MCAO mode (Figure 4 - right);

- SCAO WFS plate, holding the WFS and other tools for the SCAO mode; the description of the SCAO WFS plate is beyond the scope of this paper.

The three probes of the LOR WFS for the MCAO mode cover a patrol field of view of up to 200 arcsec diameter. The exact value of the field is under investigation, in the framework of the sky coverage analysis. Each probe covers approximately $1 / 3$ of the patrol field, with overlap.

Each LOR WFS probe is divided in two channels:

- $\quad$ Low-Order WFS channel, including a $2 \times 2$ Shack-Hartmann WFS working in the wavelength range 1.5-1.8 $\mu \mathrm{m}$;

- Reference WFS channel, including a 10×10 Shack-Hartmann WFS working in the wavelength range 0.6-1.0 $\mu \mathrm{m}$.

MAORY shall permit astrometric observations with extreme relative accuracy of 50 micro-arcsec over short and long timescales, of up to 5 years. Positioning errors of the LOR WFS probes are propagated through the MCAO system translating into field warping which can only partially be calibrated by geometric transformations of the science images. The tolerable positioning errors of the LOR WFS probes correspond to about 2 mas on sky. Of course the achievement of such extreme relative astrometric accuracy will only be possible through dedicated calibration procedures. 

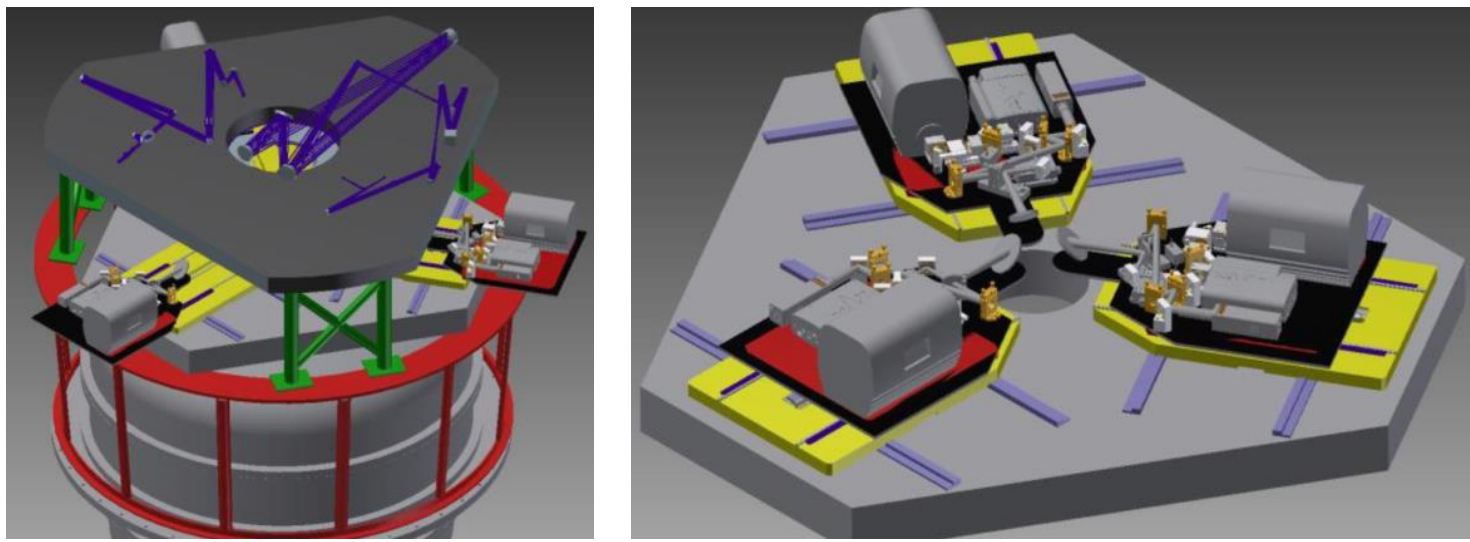

Figure 4. Left: NGS wavefront sensor module, consisting of two plates (above - SCAO plate; below - LOR plate). The module is connected to the MICADO cryostat by an interface structure (red). Right: LOR plate with 3 WFS units.

\subsection{Real time control system}

The MAORY RTC architecture ${ }^{[11]}$ and the main control loops are shown in Figure 5.

The sensors are shown on the left: from top to bottom, these are the LGS WFS, the NGS Low-Order WFS and the NGS Reference WFS. The actuators are shown on the right: from bottom to top, these are the telescope's M4 and M5 (plus other actuators which are not shown here for simplicity), the MAORY post-focal DMs (called DM1 and DM2 in the diagram) and the LGS jitter compensation mirrors (JM), which could be inside the LGS WFS probes or directly in the LGS launch telescopes (the trade-off is in progress).

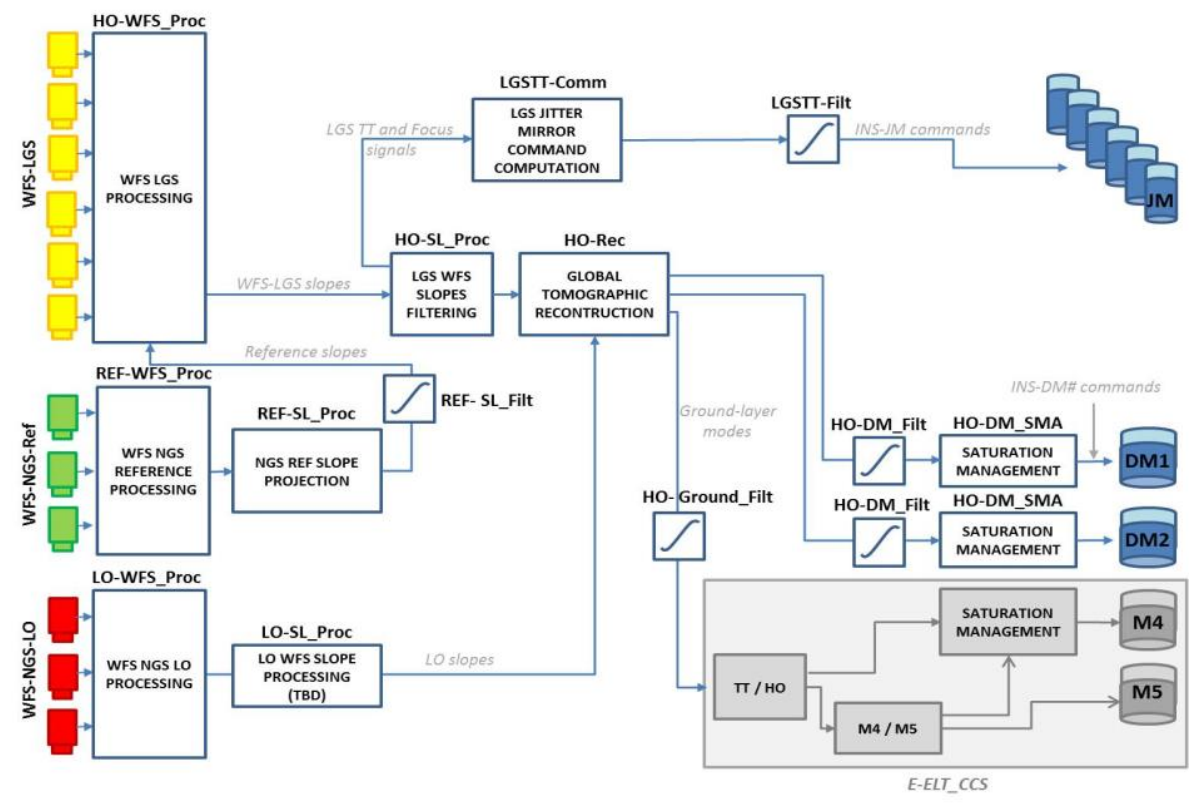

Figure 5. Real time control system architecture.

The LGS WFS images are calibrated and processed and the slopes are computed. The slopes are filtered from the signal corresponding to tip-tilt, focus and astigmatism. The tip-tilt signal is used to drive the LGS jitter compensation loop, which is shown in the upper part of the diagram, while the focus signal is used for re-focusing the LGS WFS probes (this 
loop is not shown in the diagram). The filtered slopes are used to populate the slope vector, which is fed into the global tomographic reconstructor. In the simplest approach, the reconstructor is based on a Matrix-Vector-Multiply (MVM), where the control matrix is computed as pseudo-inverse of the WFSs-to-DMs interaction matrix, e.g. by truncated singular value decomposition. Also a more advanced POLC reconstructor ${ }^{[12]}$ is considered. The baseline frame rate of the LGS WFS high-order loop is $500 \mathrm{~Hz}$.

The NGS Reference WFS images are calibrated and processed and the slopes are computed and projected in the directions of the 6 LGSs, to be used as slope offsets. The frame rate of the NGS Reference WFS loop is slow, typically 0.1-10 Hz.

The NGS Low-Order WFS images are calibrated and processed and the slopes (corresponding to the tip-tilt, focus and astigmatism modes) are used to populate the slope vector, which is fed into the global tomographic reconstructor, along with the high-order slopes from the LGS WFS. The frame rate of the NGS Low-Order WFS loop ranges from a minimum of $200 \mathrm{~Hz}$ to a maximum of $1000 \mathrm{~Hz}$, also depending on the NGS brightness.

The commands produced by the tomographic reconstructor are temporally filtered and sent to the DMs. In the case of M4/M5, the commands are sent to the telescope CCS, which takes care of tip-tilt / high-order splitting between the actuators in the telescope.

\subsection{Wavefront error budget and MCAO performance}

The optimisation of the MCAO architecture and the related performance estimation are carried by end-to-end simulation tools ${ }^{[13][14]}$

In MCAO mode, MAORY will have to provide Strehl Ratio $\mathrm{SR} \geq 0.3$ at wavelength $\lambda=2.2 \mu \mathrm{m}$ under median atmospheric conditions $(\mathrm{r} 0=0.157$ at wavelength $\lambda=0.5 \mu \mathrm{m})$. This requirement is intended as average value over the MICADO field of view for observations as close to zenith as allowed by the telescope. The requirement has to be achieved over at least $50 \%$ of the sky observable by the telescope, at any Galactic latitude. The performance goal, for the same conditions, is $\mathrm{SR}=0.5$.

Table 3 shows the high-level wavefront error budget breakdown. This error budget is on-axis, but very little variation is expected over the MICADO scientific field of view. The main contributors are the telescope itself and the MCAO architecture, including the number of LGS and NGS, the number of sub-apertures in the WFSs, the number and spacing of actuators in the DMs, the DMs conjugation ranges. The RTC wavefront error is for a POLC reconstructor.

Table 3. Wavefront error budget for median seeing conditions and two post-focal DMs in MAORY.

\begin{tabular}{|l|r|}
\hline Sub-system / item & RMS wavefront error [nm] \\
\hline ELT & 145 \\
\hline MCAO architecture & 217 \\
\hline Post-focal relay optics & 67 \\
\hline NGS WFS & 99 \\
\hline LGS WFS & 98 \\
\hline RTC & 87 \\
\hline Contingency & 48 \\
\hline Total & $\mathbf{3 1 9}$ \\
\hline
\end{tabular}

The overall wavefront error $(319 \mathrm{~nm})$ corresponds to $\mathrm{SR}=0.44$ at $\lambda=2.2 \mu \mathrm{m}$, which is well above the requirement, but anyway below the goal. The $50 \%$ sky coverage requirement is still under assessment.

The table refers in particular to the MAORY configuration with two DMs in the MCAO module, in addition to the telescope's M4/M5. The main advantages of this configuration, with respect to a simplified configuration with one DM only in MAORY, are: i) improved MCAO performance on the NGS patrol field allows the use of fainter NGSs, 
translating into improved sky coverage; ii) one more DM sampling the turbulence profile makes MAORY more robust to $\mathrm{Cn} 2$ profile variations; iii) one more DM ensures better performance, especially at shorter wavelengths. The number of DMs in MAORY is one of the most important on-going system trade-offs, in the framework of the project phase B.

\section{ACKNOWLEDGMENTS}

The MAORY instrument is developed in the framework of the Agreement No. 65221/ESO/15/67001/JSC between the European Organisation for Astronomical Research in the Southern Hemisphere (ESO) and the Istituto Nazionale di Astrofisica (INAF) on behalf of the Consortium consisting of INAF and of the Institut National des Sciences de I'Univers du Centre National de la Recherche Scientifique (INSU/CNRS) acting on behalf of the Institut de Planetologie et d'Astrophysique de Grenoble (IPAG).

This work has been partly supported by "Progetto Premiale E-ELT" funded by the Italian Ministry for Education, University and Research (MIUR).

\section{REFERENCES}

[1] Diolaiti, E., Ciliegi, P., Abicca, R., Agapito, G., et al., "MAORY adaptive optics module for the E-ELT," Proceedings of the SPIE 9909, article id. 99092D 7 pp. (2016).

[2] Tamai, R., Cirasuolo, M., Gonzalez, J. C., Koehler, B., et al., "The E-ELT program status," Proceedings of the SPIE 9906, id. 99060W 13 pp. (2016).

[3] Ramsay, S., Casali, M., Cirasuolo, M., Egner, S., et al., "Progress along the E-ELT instrumentation roadmap," Proceedings of the SPIE 9908, id. 99081T 12 pp. (2016).

[4] Davies, R., Schubert, J., Hartl, M., Alves, J., et al., "MICADO: first light imager for the E-ELT," Proceedings of the SPIE 9908, id. $99081 Z 12$ pp. (2016).

[5] Bonaglia, M., et al., "Design and status of the NGS WFS of MAORY," this Conference.

[6] Schreiber, L., Diolaiti, E., Arcidiacono, C., Pfrommer, T., Holzlöhner, R., Lombini, M., Hickson, P., "Impact of sodium layer variations on the performance of the E-ELT MCAO module," Proceedings of the SPIE 9148, id. 91486Q (2014).

[7] Clénet, Y., Buey, T., Rousset, G., Gendron, E., et al., "Joint MICADO-MAORY SCAO mode: specifications, prototyping, simulations and preliminary design," Proceedings of the SPIE 9909, id. 99090A 12 pp. (2016).

[8] Lombini, M., De Rosa, A., Ciliegi, P., Cortecchia, F., et al., "Optical design of the post-focal relay of MAORY," Proceedings of the SPIE 9908, article id. 9908AB 6 pp. (2016).

[9] Patti, M., et al., "Exploring MAORY performances through tolerance analysis," this Conference.

[10]Lombini, M., et al, "Laser Guide Star Objective of MAORY," this Conference.

[11] Schreiber, L., Diolaiti, E., Arcidiacono, C., Baruffolo, A., et al., "Dimensioning the MAORY real time computer," Proceedings of the SPIE 9909, article id. 99094L 11 pp. (2016).

[12] Ellerbroek, B., Vogel, C. R., "Simulations of closed-loop wavefront reconstruction for multi-conjugate adaptive optics on giant telescopes," Proceedings of the SPIE 9909, Volume 5169, pp. 206-217 (2003).

[13] Arcidiacono, C., et al., "Status of the MAORY numerical simulation tool," this Conference.

[14] Oberti, S., et al., "MAORY design trade-off study: tomography dimensioning," this Conference. 